
\title{
$\begin{array}{ll}\text { Research Square } & \begin{array}{l}\text { Preprints are preliminary reports that have not undergone peer review. } \\ \text { They should not be considered conclusive, used to inform clinical practice, } \\ \text { or referenced by the media as validated information. }\end{array}\end{array}$
}

\section{Differences between treadmill and cycle ergometer cardiopulmonary exercise testing results in amateur triathletes and their association with body composition and body mass index}

\section{Szymon Price}

3rd Department of Internal Medicine and Cardiology, Medical University of Warsaw, Warsaw, Poland

\section{Szczepan Wiecha}

Jozef Pilsudski University of Physical Education in Warsaw Faculty in Biala Podlaska

Igor Cieśliński

Jozef Pilsudski University of Physical Education in Warsaw Faculty in Biala Podlaska

Daniel Śliż ( $\nabla$ daniel.sliz@uwm.edu.pl)

3rd Department of Internal Medicine and Cardiology, Medical University of Warsaw, Warsaw, Poland

Przemysław Seweryn Kasiak

Medical University of Warsaw

Grzegorz Gruba

Medical University of Warsaw

\section{Artur Mamcarz}

3rd Department of Internal Medicine and Cardiology, Medical University of Warsaw, Warsaw, Poland

Jacek Lach

3rd Department of Internal Medicine and Cardiology, Medical University of Warsaw, Warsaw, Poland

\section{Research Article}

Keywords: CPET, treadmill (Tr), cycle ergometry (CE), body fat (BF), fat free mass (FFM), body mass index (BMI)

Posted Date: September 29th, 2021

DOl: https://doi.org/10.21203/rs.3.rs-917362/v1

License: () (1) This work is licensed under a Creative Commons Attribution 4.0 International License. Read Full License

Version of Record: A version of this preprint was published at International Journal of Environmental Research and Public Health on March 17th, 2022. See the published version at https://doi.org/10.3390/ijerph19063557. 


\section{Abstract}

Cardiopulmonary exercise testing (CPET) is the method of choice to assess aerobic fitness. Previous research was ambiguous as to whether treadmill ( $\mathrm{Tr}$ ) and cycle ergometry (CE) results are transferrable or different between testing modalities in triathletes. The aim of this paper was to investigate the differences in $\mathrm{HR}$ and $\mathrm{VO}_{2}$ at maximum exertion and at anaerobic threshold (AT) and respiratory compensation point (RCP) and evaluate their association with body fat (BF), fat free mass (FFM), and body mass index (BMI).

143 adult $(\mathrm{n}=18$ female), amateur, Caucasian triathletes had both $\mathrm{Tr}$, and CE CPET performed. The male group was divided into $<40$ years $(n=80)$ and $>40 y e a r s(n=45)$. Body composition was measured with bioelectrical impedance before tests. Differences were evaluated using paired T-tests and associations were evaluated in males using multiple linear regression (MLR).

Significant differences were found in $\mathrm{VO}_{2}$ and $\mathrm{HR}$ at maximum exertion, at $\mathrm{AT}$ and at RCP between $\mathrm{CE}$ and Tr testing, in both males and females. $\mathrm{VO}_{2 A T}$ was $38.8( \pm 4.6) \mathrm{ml} / \mathrm{kg} / \mathrm{min}$ in $\operatorname{Tr} v s 32.8( \pm 5.4)$ in CE in males and 36.0( \pm 3.6$)$ vs 32.1 $( \pm 3.8)$ in females $(p<0.001)$. $\mathrm{HR}_{A T}$ was $149( \pm 10) \mathrm{bpm}$ in Trvs $136( \pm 11)$ in CE in males and $156( \pm 7)$ vs $146( \pm 11)$ in females $(p<0.001) . \mathrm{VO}_{2} \mathrm{max}$ was $52( \pm 6) \mathrm{ml} / \mathrm{kg} / \mathrm{min}$ vs $49( \pm 7)$ in CE in males and $45.3( \pm 4.9)$ in $\operatorname{Tr} v s 43.9( \pm 5.2)$ in females $(p<0.001)$. HRmax was $183( \pm 10)$ bpm in $\operatorname{Tr} v s 177( \pm 10)$ in CE in males and $183( \pm 9)$ vs $179( \pm 10)$ in females $(p<0.001)$. MLR showed that BMI, BF and FFM are significantly associated with differences in $\mathrm{HR}$ and $\mathrm{VO}_{2}$ at maximum, AT and $\mathrm{RCP}$ in males aged $>40$.

Both tests should be used independently to achieve optimal fitness assessment and further training planning.

\section{Introduction}

Cardiopulmonary exercise testing (CPET) is a dynamic, non-invasive method to assess the cardiopulmonary system at rest and during exercise $^{1}$. It may be applied in medicine to evaluate the degree of cardiovascular function impairment and plan rehabilitation, and in sport science to assess participants fitness ${ }^{2}$. Key variables measured in CPET include heart rate (HR), oxygen consumption $\left(\mathrm{VO}_{2}\right)$, respiratory rate (RR), pulmonary ventilation (VE), oxygen pulse, respiratory exchange ratio (RER), ventilatory equivalents for oxygen $\left(\mathrm{VE} / \mathrm{VO}_{2}\right)$ and for carbon dioxide $\left(\mathrm{VE} / \mathrm{VCO}_{2}\right)^{3}$. The most frequently compared variable is the maximum oxygen uptake $\left(\mathrm{VO}_{2} \mathrm{max}\right)$, which may be defined as the highest value reached, despite progressive increase of the load applied, with the development of a plateau in the $\mathrm{VO}_{2}{ }^{3}$. Another important value is the $\mathrm{VO}_{2}$ at the anaerobic threshold (AT), corresponding to the threshold between moderate and high intensity exercise, is the point when the lack of sufficient oxygen supply to the exercising muscles necessitates glycolytic ATP production and accumulation of lactic acid ${ }^{4}$. Thus, in exercise at intensity below AT, lactate remains at resting levels, while in high intensity exercise above ${ }_{A T}$, lactate rises until an elevated steady state is attained ${ }^{4}$. The respiratory compensation point (RCP) is identified as the second breakpoint in the ventilation response and is a measurable variable most closely related to the concept of critical power (CP), which in turn represents the point separating power outputs that can be sustained for a prolonged time from power outputs which lead to a certain maximum after which exercise intolerance occurs ${ }^{4,5}$. CP is especially relevant in high intensity training or intermittent high intensity training ${ }^{5}$.

The most commonly used testing modalities are the cycle ergometer and treadmill, used with various protocols or self-paced ${ }^{6,7}$. These training modalities both have unique strengths and weaknesses. The treadmill activates more muscle groups and $\mathrm{VO}_{2}$ max is generally higher than in the cycle ergometer by $7-18 \%$, varying between studies, although there exist many conflicting papers and results are inconsistent ${ }^{8}$. The cycle ergometer allows better electrocardiographic (ECG) analysis due to fewer artifacts from upper body motion and is safer and less intimidating for people unaccustomed with a moving treadmill ${ }^{6}$.

The relationship between testing modality and $\mathrm{VO}_{2}$ max is ambiguous. In untrained subjects, $\mathrm{VO}_{2}$ max is generally higher in treadmill testing. However, athletes with previous experience in cycling may obtain results on the cycle ergometer which are equal to or even higher than those obtained on the treadmill, while trained runners display higher results in treadmill testing ${ }^{9,10}$. Usually for athletes the testing modality is selected specifically to fit the discipline trained by the examinee, i.e. a treadmill for runners or a cycle ergometer for cyclists ${ }^{11}$. A unique challenge is posed by triathletes, who have no single mode of training, but rather devote a portion of their training to swimming, cycling and running ${ }^{8}$. The monitoring of training would therefore ideally be carried out with all of the specific tests for the most accurate results, but this would be highly impractical given that testing is time consuming, costly and must be repeated regularly ${ }^{11}$. It is therefore important to know whether there is a significant difference between treadmill and cycle ergometry results in triathletes. Few studies have been conducted to assess this, and existing studies often included small studied groups of fewer than twenty participants ${ }^{8}$. The results of previous studies are inconclusive, showing that $\mathrm{VO}_{2}$ max in triathletes may be equal ${ }^{12-16}$, higher ${ }^{17,18}$ or even lower ${ }^{19}$ in treadmill 
testing compared with cycle ergometry. The anaerobic threshold (AT) and lactate threshold (LT) were also either reported as higher in the treadmill test ${ }^{20}$, or similar in both tests ${ }^{21}$. Millet et al. point out that study methods were often unclear and the study group sizes were limited in many of the existing studies ${ }^{8}$.

Maximal heart rate (HRmax) is either reported as similar $14,17,20,22$, or slightly higher in treadmill testing compared with cycle ergometry $19,20,23$. It is also unclear whether this relationship is true for males and females alike, or only for males ${ }^{8}$. Another important parameter to consider is the HR corresponding to the AT, which is often used to prescribe submaximal exercise training loads ${ }^{8}$. This value has previously been generally reported as higher in treadmill tests compared with cycle ergometry ${ }^{14,19,23-25}$. However, some studies yielded no significant difference ${ }^{12,26}$.

The sex differences between triathletes in running and cycling are also unclear. Most studies did not evaluate these differences at all and the ones that did yielded no difference between cycling and running for both males and females ${ }^{15,27}$.

It has been proposed that the difference in $\mathrm{VO}_{2}$ max between treadmill and cycle ergometry varies between runners and triathletes may be due to the higher muscle mass of triathletes, especially in the upper body, and not to running economy ${ }^{8}$. To the best of our knowledge, no studies evaluated whether an association exists between body composition (BC) and differences in $\mathrm{VO}_{2}$ max in different testing modalities in triathletes.

The main aim of this study was to assess the difference in $\mathrm{VO}_{2}$ and $\mathrm{HR}$ at maximum exertion and at AT and RCP in cycle ergometry and treadmill testing. A further aim was to evaluate whether an association exists between these parameters and BC or body mass index (BMI).

\section{Materials And Methods}

The data of participants was gathered from records of commercial CPET performed in years 2013-2020. They were recruited via internet and social media advertisements, or via recommendation from trainers or other clients. The tests were carried out on personal request of the participants as part of training optimization and diagnostics. The participants were amateur triathletes who had participated in competitive events. Inclusion criteria for the study were: age over 18 years, triathlon training for at least 3 months, having a treadmill test and a cycle ergometer test performed within a maximum 2 months' timeframe, and meeting the maximum exertion criteria described below. Exclusion criteria were any chronic or acute medical conditions (including musculoskeletal system disorders like new fractures and sprains, as well as addiction to nicotine, alcohol, or other substances) or ongoing intake of any medication. Identical study methods and procedures were used during the entire period from which data were gathered. Participants were informed via e-mail on how to prepare for the test. They were advised to avoid any exercise $2 \mathrm{~h}$ prior to the test, eat a light carbohydrate meal $2-3 \mathrm{~h}$ before the test and stay hydrated by drinking isotonic beverages. They were also instructed to avoid medicines, caffeine, and cigarettes before the test.

From the database, we obtained 238 individual cases of people who carried out the study twice (cycling and running separately). After verifying the inclusion criteria, we obtained 143 cases included in further analysis. Populational data was calculated as means with standard deviation (SD) and is presented in Table 1. Body mass (BM) and fat mass (FM) were measured with the use of a BC analyzer (Tanita, MC 718, Japan) before every test with the multifrequency $5 \mathrm{kHz} / 50 \mathrm{kHz} / 250 \mathrm{kHz}$ electrical bioimpedance method. The BC tests were conducted directly prior to each CPET if the interval between tests was $>48 \mathrm{~h}$ and mean values from both tests were further analyzed. In cases where two CPET tests were carried out on following days, only one BC analysis was performed prior to the first CPET. All measurements (BC and CPET) took place under similar conditions in the medical clinic Sportslab (www.sportslab.pl, Warsaw, Poland). The conditions were: $40 \mathrm{~m}^{2}$ of indoor, air-conditioned space, altitude $100 \mathrm{~m} \mathrm{MSL}$, temperature 20-22 degrees Centigrade, 40-60\% humidity. Exercise tests were performed on a cycle ergometer Cyclus-2 (RBM elektronik-automation GmbH, Leipzig, Germany) and on a mechanical treadmill (h/p/Cosmos quasar, Germany), within 1day - 2months of one another. 
Table 1

Population characteristics for males and females, including characteristics of the age groups $\leq 40$ and $>40$ years of age in males and the mean differences between them.

\begin{tabular}{|c|c|c|c|c|c|c|}
\hline \multicolumn{7}{|c|}{ Female athletes } \\
\hline Characteristic & \multicolumn{6}{|l|}{ All $n=18^{1}$} \\
\hline Age & \multicolumn{6}{|l|}{$33(7)$} \\
\hline Height & \multicolumn{6}{|l|}{$169(4)$} \\
\hline Weight & \multicolumn{6}{|l|}{$61.9(4.4)$} \\
\hline BMI & \multicolumn{6}{|l|}{$21.70(1.37)$} \\
\hline $\mathrm{BF}$ & \multicolumn{6}{|l|}{$23.3(3.5)$} \\
\hline FM & \multicolumn{6}{|l|}{$14.55(2.93)$} \\
\hline FFM & \multicolumn{6}{|l|}{$47.38(2.61)$} \\
\hline \multicolumn{7}{|l|}{ Male athletes } \\
\hline Characteristic & All $n=125$ & $\leq 40, n=80^{1}$ & $>40, n=45^{1}$ & Difference $^{2}$ & $95 \% \mathrm{Cl}^{2,3}$ & p-value ${ }^{2}$ \\
\hline Age & $38(10)$ & $32(5)$ & $46(8)$ & 15.39 & $12.10-15.67$ & $<0.001$ \\
\hline Height & $181(7)$ & $181.0(7.0)$ & $180.0(6.0)$ & 1.40 & $-1.00,3.80$ & 0.3 \\
\hline Weight & $79(9)$ & $78.0(10.0)$ & $79.0(8.0)$ & -0.67 & $-4.00,2.70$ & 0.7 \\
\hline BMI & $24.04(2.19)$ & $23.8(2.4)$ & $24.4(1.7)$ & -0.56 & $-1.30,0.18$ & 0.13 \\
\hline BF & $15.4(4.2)$ & $15.2(4.2)$ & $15.8(4.3)$ & -0.60 & $-2.20,1.00$ & 0.5 \\
\hline FM & $12.3(4.6)$ & $12.2(4.8)$ & $12.6(4.1)$ & -0.47 & $-2.10,1.10$ & 0.6 \\
\hline FFM & $66(6)$ & $66.0(6.0)$ & $66.0(6.0)$ & -0.21 & $-2.40,2.00$ & 0.9 \\
\hline \multicolumn{7}{|l|}{${ }^{1}$ Mean (SD) } \\
\hline \multicolumn{7}{|c|}{${ }^{2}$ Welch Two Sample t-test } \\
\hline \multicolumn{7}{|c|}{${ }^{3} \mathrm{Cl}=$ Confidence Interval } \\
\hline \multicolumn{7}{|c|}{ Height $(\mathrm{cm})$; Weight $(\mathrm{kg})$; BMl, body masa index; $\mathrm{BF}$, body fat (\%); FM, fat mass $(\mathrm{kg})$; FFM, fat free mass $(\mathrm{kg})$} \\
\hline
\end{tabular}

During all tests, cardio-pulmonary indices were recorded using a Cosmed Quark CPET device (Rome, Italy), calibrated before each test according to the manufacturer's instructions. HR was measured using the ANT + chest strap which is part of the Cosmed Quark CPET device (declared accuracy similar to ECG $\pm 1 \mathrm{bpm}$.). Each test was preceded by a $5 \mathrm{~min}$ warm up (walking or pedaling with no resistance). To account for different exercise capacity of the athletes, the initial power (Watt) or speed $(\mathrm{km} / \mathrm{h}$ ) were determined based on an interview carried out before each individual test. The lowest power at which the participant subjectively felt resistance was selected as the initial power for cycle ergometer tests (60-150W). The power was then increased by $20-30 \mathrm{~W}$ every $2 \mathrm{~min}$. For treadmill tests the start speed was an individually selected slow running pace, between 7 and $12 \mathrm{~km} / \mathrm{h}$ based on interview and $1 \%$ incline was applied. The speed was then increased by $1 \mathrm{~km} / \mathrm{h}$ every $2 \mathrm{~min}$. To assess the maximum level of aerobic fitness, participants were instructed to maintain the effort for as long as possible, encouraged verbally to the greatest possible effort. They could terminate the test at any moment if they felt they could no longer maintain the exertion level. Participants were under cardiopulmonary monitoring during the entire test. Before each CPET, after each change of load, and 3 minutes after the test, $20 \mu \mathrm{L}$ of blood were collected from the fingertip for determination of lactate concentration (LA) using the Super GL2 analyzer (Müller Gerätebau GmbH, Freital, Germany) calibrated before each series of samples. There were no interruptions in the CPET during the collection of blood samples. During the running test, the athlete, while running, put his hand on the rail attached to the treadmill and a technician took a blood sample. Before the sample was drawn into the capillary, the first drops of blood were carefully squeezed into a swab. Similarly, during the cycling test, the subject was asked to relax their hands for about 20-30 seconds before the collection, and then the first drops of blood were discarded before taking the sample into a capillary. 
The test was terminated by the operator if either $\mathrm{VO}_{2}$ or $\mathrm{HR}$ showed no further increase with increasing speed/power. The results of the $\mathrm{BC}$ analysis and CPET were saved as an Excel (Microsoft corporation, Washington, United States) spreadsheet for further analysis. The raw data were anonymized and processed with the use of a custom program created in Python software to identify data at AT, RCP, and maximum exertion. In accordance with current standards, CPET data were recorded breath by breath and then averaged across 15-s intervals; the highest HR in the interval was recoded and HR values were not averaged ${ }^{28}$. For statistical evaluation we included only cases where 3 of 4 following criteria were met: RER during test reaching $>1.10, \mathrm{VO}_{2}$ plateau (an increase in $\mathrm{VO}_{2}$ with increasing speed/power lower than $100 \mathrm{ml} / \mathrm{min}$ ), respiratory frequency over 45/min, perceived exertion over 18 in Borg scale ${ }^{29}$. AT and RCP were located from visual inspection. It was assumed that AT was reached after the following criteria were met: (1) $\mathrm{VE} \mathrm{VO}_{2} \mathrm{Curve}$ begins to rise with constant VE/ $\mathrm{VCO}_{2}$ curve; (2) End-Tidal Partial Pressure of Oxygen begins to rise with constant End-Tidal Partial Pressure of Carbon Dioxide 30.

It was assumed that RCP was reached after the following criteria were met: (1) a decrease in PetCO ${ }_{2}$ after reaching maximal level; (2) a rapid nonlinear increase in VE (second deflection); (3) the $\mathrm{VE} / \mathrm{VCO}_{2}$ ratio reached a minimum and began to increase; (4) a nonlinear increase in $\mathrm{VCO}_{2}$ versus $\mathrm{VO}_{2}$ (departure from linearity) ${ }^{30}$.

Retrospective performance data

Competition experience was assessed using the enduhub.com database (Enduhub Corporation, Newark, DE). It is a commonly available website where official scores of participants' competitions on standardized distances (1/8, 1/4, 1/2 Ironman, Sprinter and Olympic distances of triathlon were included) are uploaded by event organizers. Each score in this database is thoroughly validated by professional companies specialized in time measuring during sports events (Datasport, Szczawno Zdrój, Poland and STS-Timing, Łubianka, Poland. Results are verified before publication by enduhub.com editors.

We used the earliest officially available score from distance-standardized triathlon and used it as a starting point to assess competition experience which was presented in months (month of competition and CPET were both included). These times were applied to calculate how long each sportsman is engaged in regular training and actively take part in public competitions.

\section{Ethical considerations}

The study involving human participants was reviewed and approved by the Bioethical Committee of the Medical University of Warsaw. The patients/participants provided their written informed consent to participate in this study. All procedures were carried out in accordance with the Declaration of Helsinki.

\section{Data analysis}

Statistical analysis has been conducted in R environment/programming language for statistical computing (version 3.6.4) and Imtest and gtsummary libraries ${ }^{31,32}$.

Missing data were identified in lactate values in 7 cases and imputation was performed with random forests ${ }^{33}$.

Normality was tested with the Anderson-Darling test. Data were calculated as means with SD and 95\% confidence intervals (CI). Differences between results of both testing modalities were calculated using paired T-tests. A significance level of $p<0.05$ was adopted for all results.

MLR models were created to evaluate the relationship between the differences in results from treadmill and cycle ergometry (dependent variables), and body fat (BF), fat free mass (FFM), and BMI. Several regression models were initially tested and MLR was selected as the best fit based on the Akaike information criterion. The models were only created for the male population due to group sizes. The population was also divided according to age into two groups, $<40$ years and $>40$ years (age was not included as an independent variable in these models). The division was based on the assumption that aerobic capacity decreases rapidly after the age of 40 years in males and is related to muscle mass ${ }^{34,35}$. The Harvey- Collier test was used to test linearity. R-squared $\left(R^{2}\right)$ was used to assess the quality of the models.

\section{Results}

The differences between CPET results in cycle ergometry and treadmill testing are presented in Table 2 for females and Tables $3-5$ for males. Results of MLR are presented in Table 6. Only regression results in the two age subgroups are presented, as no significant 
relationships were found in the whole male population. All statistically significant results are marked bold. Selected (based on highest $\mathrm{R}^{2}$ ) relationships are presented as linear regression graphs (Fig. 1). 
Table 2

Differences between cycle ergometry (CE) and treadmill (Tr) CPET results in female participants; significant results are bold.

\begin{tabular}{|c|c|c|c|c|c|}
\hline Characteristic & $C E, n=18^{1}$ & $\operatorname{Tr}, \mathrm{n}=18^{1}$ & Difference $^{2}$ & $95 \% \mathrm{Cl}^{2,3}$ & p-value ${ }^{2}$ \\
\hline $\mathrm{VO}_{2 A T}$ & 32.1 (3.8) & $36.0(3.6)$ & -3.9 & $-5.4,-2.5$ & $<0.001$ \\
\hline $\mathrm{VO}_{2 \mathrm{ATa}}$ & $1,976(257)$ & $2,216(221)$ & -240 & $-328,-152$ & $<0.001$ \\
\hline $\mathrm{RER}_{\mathrm{AT}}$ & $0.87(0.05)$ & $0.88(0.03)$ & -0.01 & $-0.04,0.02$ & 0.5 \\
\hline $\mathrm{HR}_{\mathrm{AT}}$ & $146(11)$ & $156(7)$ & -10 & $-14,-6.1$ & $<0.001$ \\
\hline $\mathrm{O}_{2}$ pulse $_{\mathrm{AT}}$ & $13.64(2.07)$ & $14.28(1.84)$ & -0.65 & $-1.2,-0.07$ & 0.031 \\
\hline $\mathrm{VE}_{\mathrm{AT}}$ & $53(7)$ & $62(8)$ & -8.5 & $-12,-4.4$ & $<0.001$ \\
\hline $\mathrm{RR}_{\mathrm{AT}}$ & $29(5)$ & $36(8)$ & -6.7 & $-9.5,-3.9$ & $<0.001$ \\
\hline $\operatorname{Lac}_{A T}$ & $1.77(0.54)$ & $1.87(0.46)$ & -0.11 & $-0.32,0.10$ & 0.3 \\
\hline $\mathrm{VO}_{2 \mathrm{RCP}}$ & $40.2(4.5)$ & $42.9(4.7)$ & -2.8 & $-4.1,-1.4$ & $<0.001$ \\
\hline $\mathrm{VO}_{2 \mathrm{RCPa}}$ & $2,471(279)$ & $2,642(283)$ & -170 & $-255,-85$ & $<0.001$ \\
\hline $\mathrm{VCO}_{2 \mathrm{RCP}}$ & $2,468(282)$ & $2,643(283)$ & -174 & $-262,-87$ & $<0.001$ \\
\hline $\mathrm{HR}_{\mathrm{RCP}}$ & $169(9)$ & $175(8)$ & -6.7 & $-8.3,-5.1$ & $<0.001$ \\
\hline $\mathrm{VE}_{\mathrm{RCP}}$ & $80(10)$ & $86(9)$ & -5.3 & $-11,0.32$ & 0.063 \\
\hline $\mathrm{RR}_{\mathrm{RCP}}$ & $38(6)$ & $44(7)$ & -5.7 & $-7.7,-3.6$ & $<0.001$ \\
\hline $\mathrm{O}_{2}$ pulse $_{\mathrm{RCP}}$ & $14.69(1.80)$ & $15.13(1.98)$ & -0.44 & $-0.82,-0.05$ & 0.029 \\
\hline $\mathrm{Lac}_{\mathrm{RCP}}$ & 4.30 (0.69) & $4.50(0.82)$ & -0.20 & $-0.72,0.31$ & 0.4 \\
\hline $\mathrm{VO}_{2} \max$ & $43.9(5.2)$ & $45.3(4.9)$ & -1.4 & $-2.8,-0.02$ & 0.047 \\
\hline $\mathrm{VO}_{2} \max _{\mathrm{a}}$ & 2,704 (319) & $2,791(305)$ & -87 & $-169,-4.2$ & 0.040 \\
\hline RERmax & $1.13(0.03)$ & $1.11(0.03)$ & 0.01 & $-0.01,0.04$ & 0.3 \\
\hline $\mathrm{O}_{2}$ pulse $_{\max }$ & $15.12(1.96)$ & 15.33 (2.03) & -0.20 & $-0.59,0.18$ & 0.3 \\
\hline HRmax & $179(10)$ & $183(9)$ & -3.5 & $-5.2,-1.8$ & $<0.001$ \\
\hline VEmax & $110(18)$ & $104(13)$ & 6.0 & $-1.8,14$ & 0.12 \\
\hline RRmax & $55(11)$ & $55(9)$ & -0.11 & $-2.4,2.2$ & $>0.9$ \\
\hline Lacmax & $10.76(1.94)$ & $9.40(1.28)$ & 1.4 & $0.47,2.2$ & 0.005 \\
\hline
\end{tabular}




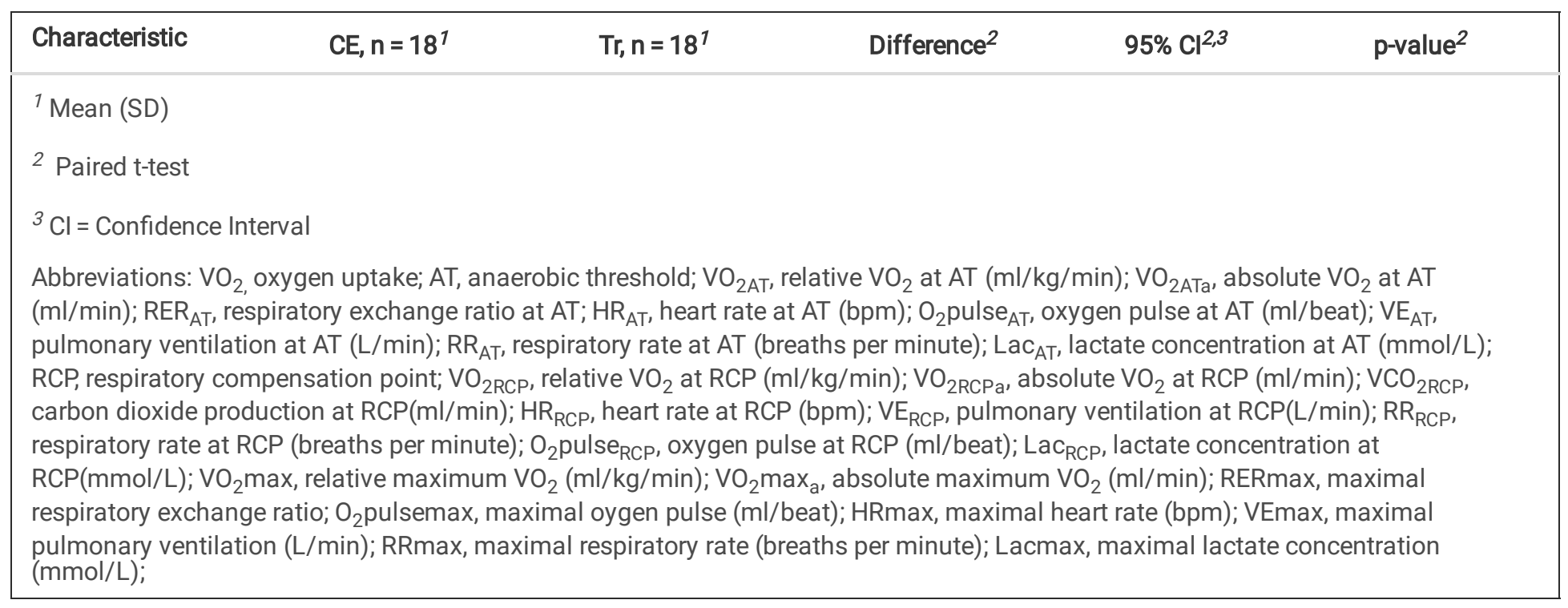


Table 3

Differences between cycle ergometry (CE) and treadmill (Tr) CPET results in male participants; significant results are bold.

\begin{tabular}{|c|c|c|c|c|c|}
\hline Characteristic & $C E, n=125^{1}$ & $\mathrm{Tr}, \mathrm{n}=125^{1}$ & Difference $^{2}$ & $95 \% \mathrm{Cl}^{2,3}$ & p-value ${ }^{2}$ \\
\hline $\mathrm{VO}_{2 \mathrm{AT}}$ & 32.8 (5.4) & $38.8(4.6)$ & -6.0 & $-6.7,-5.3$ & $<0.001$ \\
\hline $\mathrm{VO}_{2 \mathrm{ATa}}$ & $2,530(366)$ & $3,012(345)$ & -482 & $-541,-424$ & $<0.001$ \\
\hline $\mathrm{RER}_{\mathrm{AT}}$ & $0.86(0.06)$ & $0.89(0.04)$ & -0.02 & $-0.04,-0.01$ & $<0.001$ \\
\hline $\mathrm{HR}_{\mathrm{AT}}$ & $136(11)$ & $149(10)$ & -13 & $-15,-11$ & $<0.001$ \\
\hline $\mathrm{O}_{2}$ pulse $_{\mathrm{AT}}$ & $18.73(2.88)$ & $20.32(2.36)$ & -1.6 & $-1.9,-1.2$ & $<0.001$ \\
\hline $\mathrm{VE}_{\mathrm{AT}}$ & $66(10)$ & $83(11)$ & -17 & $-18,-15$ & $<0.001$ \\
\hline $\mathrm{RR}_{\mathrm{AT}}$ & $28(5)$ & $37(8)$ & -8.2 & $-9.3,-7.1$ & $<0.001$ \\
\hline $\operatorname{Lac}_{A T}$ & $1.70(0.37)$ & $1.77(0.40)$ & -0.07 & $-0.14,0.00$ & 0.043 \\
\hline $\mathrm{VO}_{2 \mathrm{RCP}}$ & $44(8)$ & $48(6)$ & -4.1 & $-5.1,-3.1$ & $<0.001$ \\
\hline $\mathrm{VO}_{2 \mathrm{RCPa}}$ & 3,372 (533) & $3,707(420)$ & -335 & $-411,-259$ & $<0.001$ \\
\hline $\mathrm{VCO}_{2 \mathrm{RCP}}$ & 3,378 (539) & $3,708(420)$ & -329 & $-406,-252$ & $<0.001$ \\
\hline $\mathrm{HR}_{\mathrm{RCP}}$ & $163(10)$ & $172(9)$ & -9.4 & $-11,-8.2$ & $<0.001$ \\
\hline $\mathrm{VE}_{\mathrm{RCP}}$ & 110 (17) & 119 (15) & -9.4 & $-12,-6.9$ & $<0.001$ \\
\hline $\mathrm{RR}_{\mathrm{RCP}}$ & $39(7)$ & $46(10)$ & -6.7 & $-8.1,-5.2$ & $<0.001$ \\
\hline $\mathrm{O}_{2}$ pulse $_{\mathrm{RCP}}$ & $20.78(3.44)$ & $21.59(2.65)$ & -0.81 & $-1.2,-0.39$ & $<0.001$ \\
\hline $\mathrm{Lac}_{\mathrm{RCP}}$ & $4.21(0.60)$ & $4.29(0.74)$ & -0.08 & $-0.23,0.06$ & 0.3 \\
\hline $\mathrm{VO}_{2} \max$ & $49(7)$ & $52(6)$ & -2.9 & $-3.6,-2.1$ & $<0.001$ \\
\hline $\mathrm{vO}_{2} \max _{\mathrm{a}}$ & $3,808(476)$ & $4,045(435)$ & -238 & $-300,-175$ & $<0.001$ \\
\hline RERmax & $1.13(0.04)$ & $1.11(0.03)$ & 0.02 & $0.02,0.03$ & $<0.001$ \\
\hline $\mathrm{O}_{2}$ pulse $\max _{\max }$ & $21.54(2.91)$ & $22.16(2.66)$ & -0.62 & $-0.94,-0.29$ & $<0.001$ \\
\hline HRmax & 177 (10) & $183(10)$ & -5.9 & $-6.8,-5.0$ & $<0.001$ \\
\hline VEmax & $158(24)$ & $152(18)$ & 6.6 & $3.4,9.8$ & $<0.001$ \\
\hline RRmax & $57(10)$ & $58(10)$ & -1.8 & $-3.6,-0.05$ & 0.044 \\
\hline Lacmax & $10.93(1.82)$ & $9.68(1.65)$ & 1.2 & $0.91,1.6$ & $<0.001$ \\
\hline
\end{tabular}




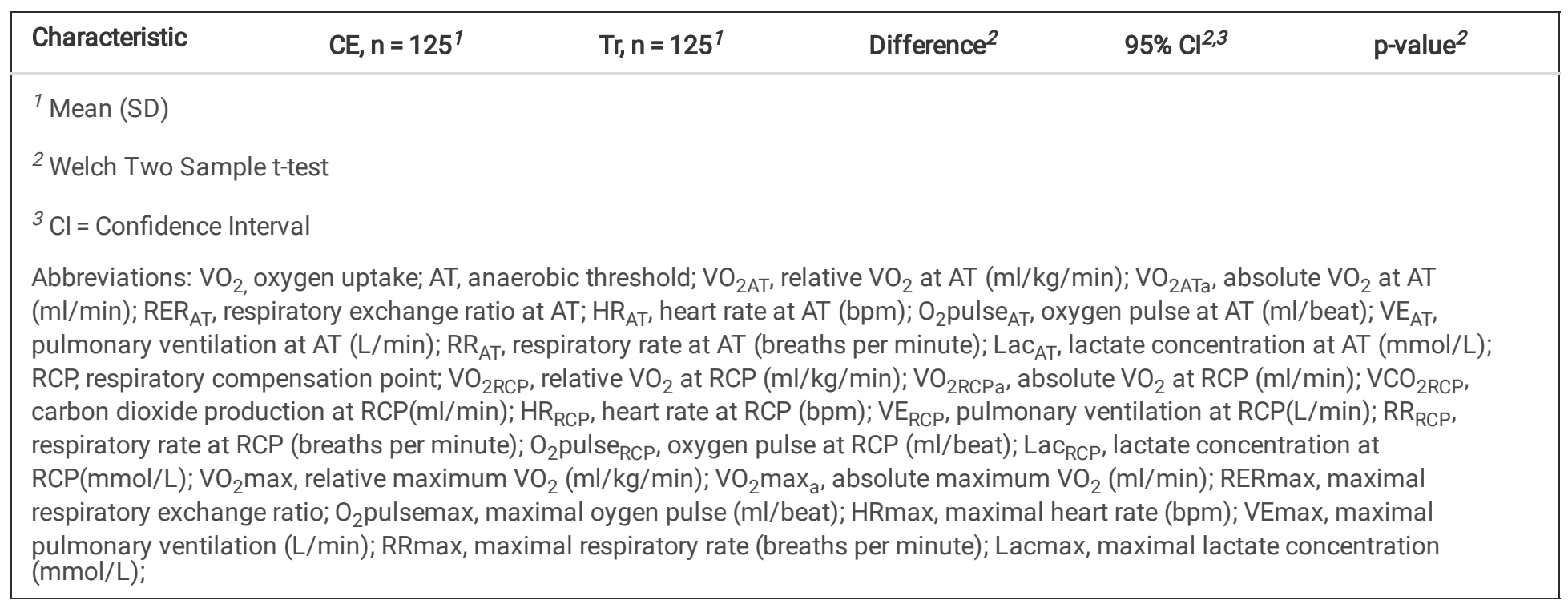


Table 4

Differences between cycle ergometry (CE) and treadmill ( $T r$ ) CPET results in male participants $\leq 40$ years; significant results are bold.

\begin{tabular}{|c|c|c|c|c|c|}
\hline Characteristic & $\mathrm{CE}, \mathrm{n}=80^{1}$ & $\operatorname{Tr}, \mathrm{n}=80^{1}$ & Difference $^{2}$ & $95 \% \mathrm{Cl}^{2,3}$ & p-value ${ }^{2}$ \\
\hline $\mathrm{VO}_{2 \mathrm{AT}}$ & $34(6)$ & $40(5)$ & -6.2 & $-7.2,-5.1$ & $<0.001$ \\
\hline $\mathrm{VO}_{2 \mathrm{ATa}}$ & $2,584(363)$ & $3,078(350)$ & -494 & $-577,-412$ & $<0.001$ \\
\hline $\mathrm{RER}_{\mathrm{AT}}$ & $0.87(0.06)$ & $0.88(0.04)$ & -0.02 & $-0.03,0.00$ & 0.047 \\
\hline $\mathrm{HR}_{\mathrm{AT}}$ & $140(11)$ & $151(10)$ & -12 & $-14,-9.5$ & $<0.001$ \\
\hline $\mathrm{O}_{2}$ pulse $_{\mathrm{AT}}$ & $18.60(2.86)$ & $20.40(2.41)$ & -1.8 & $-2.3,-1.3$ & $<0.001$ \\
\hline $\mathrm{VE}_{\mathrm{AT}}$ & $66(10)$ & $82(11)$ & -17 & $-19,-14$ & $<0.001$ \\
\hline $\mathrm{RR}_{\mathrm{AT}}$ & $28(6)$ & $36(7)$ & -8.2 & $-9.5,-6.9$ & $<0.001$ \\
\hline $\operatorname{Lac}_{A T}$ & $1.67(0.37)$ & $1.76(0.40)$ & -0.08 & $-0.17,0.00$ & 0.057 \\
\hline $\mathrm{VO}_{2 \mathrm{RCP}}$ & $45(7)$ & $49(6)$ & -4.0 & $-5.0,-2.9$ & $<0.001$ \\
\hline $\mathrm{VO}_{2 \mathrm{RCPa}}$ & 3,461 (447) & $3,790(417)$ & -328 & $-415,-242$ & $<0.001$ \\
\hline $\mathrm{VCO}_{2 \mathrm{RCP}}$ & $3,463(458)$ & $3,791(417)$ & -328 & $-417,-239$ & $<0.001$ \\
\hline $\mathrm{HR}_{\mathrm{RCP}}$ & $166(10)$ & $175(9)$ & -8.9 & $-10,-7.4$ & $<0.001$ \\
\hline $\mathrm{VE}_{\mathrm{RCP}}$ & $109(16)$ & 119 (15) & -10 & $-13,-7.0$ & $<0.001$ \\
\hline $\mathrm{RR}_{\mathrm{RCP}}$ & $38(7)$ & $45(9)$ & -6.4 & $-8.0,-4.9$ & $<0.001$ \\
\hline $\mathrm{O}_{2}$ pulse RCP & $20.90(2.94)$ & $21.71(2.69)$ & -0.81 & $-1.3,-0.36$ & $<0.001$ \\
\hline $\mathrm{Lac}_{\mathrm{RCP}}$ & $4.15(0.63)$ & $4.24(0.72)$ & -0.08 & $-0.26,0.10$ & 0.4 \\
\hline $\mathrm{VO}_{2} \max$ & $51(7)$ & $53(7)$ & -2.8 & $-3.8,-1.7$ & $<0.001$ \\
\hline $\mathrm{vO}_{2} \max _{\mathrm{a}}$ & $3,885(471)$ & $4,118(418)$ & -233 & $-316,-149$ & $<0.001$ \\
\hline RERmax & $1.13(0.04)$ & $1.11(0.03)$ & 0.02 & $0.01,0.03$ & $<0.001$ \\
\hline $\mathrm{O}_{2}$ pulse $\max _{\max }$ & $21.65(2.94)$ & $22.22(2.62)$ & -0.57 & $-1.0,-0.14$ & 0.010 \\
\hline HRmax & $180(9)$ & $186(9)$ & -5.9 & $-7.0,-4.8$ & $<0.001$ \\
\hline VEmax & $159(24)$ & $152(16)$ & 7.3 & $2.9,12$ & 0.001 \\
\hline RRmax & $56(11)$ & $58(8)$ & -1.3 & $-3.3,0.69$ & 0.2 \\
\hline Lacmax & $10.94(1.91)$ & $9.81(1.53)$ & 1.1 & $0.59,1.7$ & $<0.001$ \\
\hline
\end{tabular}




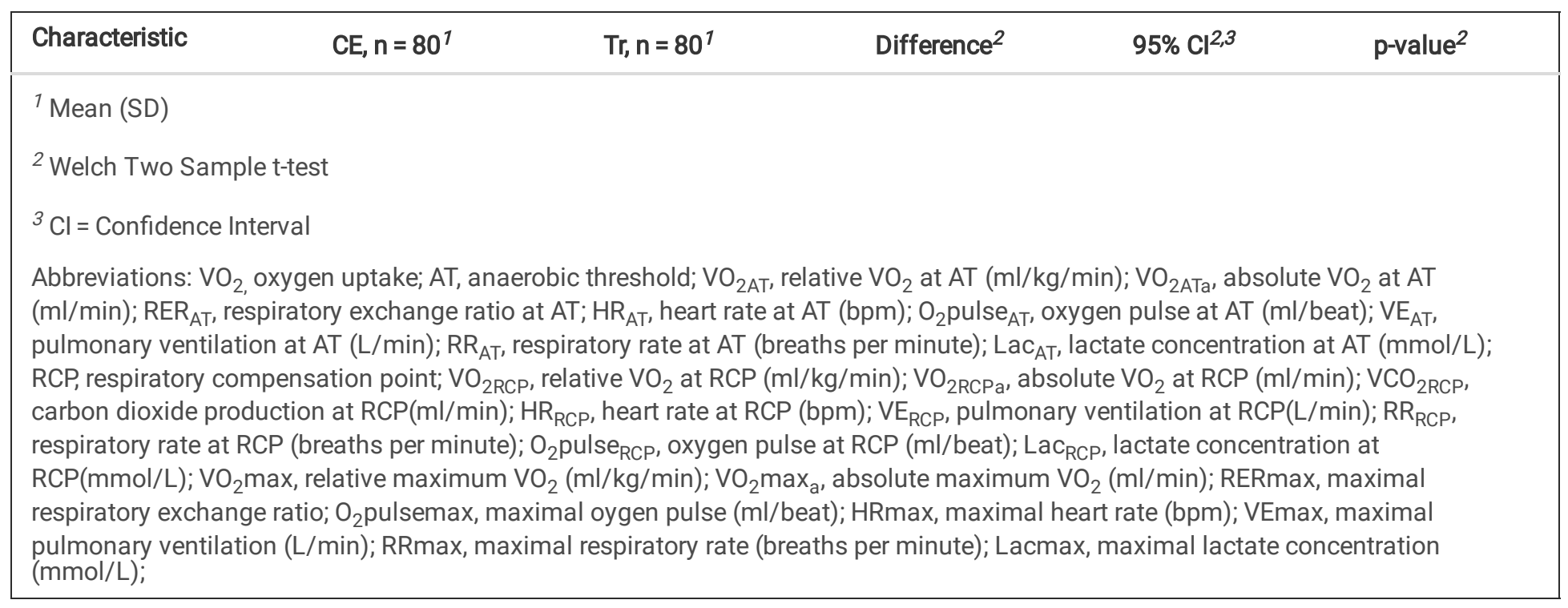


Table 5

Differences between cycle ergometry (CE) and treadmill (Tr) CPET results in male participants $>40$ years; significant results are bold.

\begin{tabular}{|c|c|c|c|c|c|}
\hline Characteristic & $C E, n=45^{1}$ & $\operatorname{Tr}, \mathrm{n}=45^{1}$ & Difference $^{2}$ & $95 \% \mathrm{Cl}^{2,3}$ & p-value ${ }^{2}$ \\
\hline $\mathrm{VO}_{2 \mathrm{AT}}$ & $31.1(4.6)$ & $36.9(3.1)$ & -5.8 & $-6.6,-4.9$ & $<0.001$ \\
\hline $\mathrm{VO}_{2 \mathrm{ATa}}$ & $2,434(354)$ & $2,895(305)$ & -461 & $-532,-390$ & $<0.001$ \\
\hline $\mathrm{RER}_{\mathrm{AT}}$ & $0.86(0.05)$ & $0.89(0.04)$ & -0.03 & $-0.05,-0.02$ & $<0.001$ \\
\hline $\mathrm{HR}_{\mathrm{AT}}$ & $129(10)$ & $144(9)$ & -15 & $-17,-12$ & $<0.001$ \\
\hline $\mathrm{O}_{2}$ pulse $_{A T}$ & $18.96(2.93)$ & $20.18(2.29)$ & -1.2 & $-1.7,-0.75$ & $<0.001$ \\
\hline $\mathrm{VE}_{\mathrm{AT}}$ & $67(11)$ & $83(10)$ & -16 & $-19,-14$ & $<0.001$ \\
\hline $\mathrm{RR}_{\mathrm{AT}}$ & 29.1 (4.7) & $37.3(8.4)$ & -8.2 & $-10,-6.2$ & $<0.001$ \\
\hline $\operatorname{Lac}_{A T}$ & $1.75(0.36)$ & $1.80(0.39)$ & -0.05 & $-0.16,0.07$ & 0.4 \\
\hline $\mathrm{VO}_{2 \mathrm{RCP}}$ & $41.1(8.4)$ & $45.4(4.3)$ & -4.3 & $-6.2,-2.4$ & $<0.001$ \\
\hline $\mathrm{VO}_{2 \mathrm{RCPa}}$ & 3,213 (634) & $3,559(387)$ & -346 & $-494,-198$ & $<0.001$ \\
\hline $\mathrm{VCO}_{2 \mathrm{RCP}}$ & 3,228 (637) & $3,560(387)$ & -332 & $-481,-182$ & $<0.001$ \\
\hline $\mathrm{HR}_{\mathrm{RCP}}$ & $157(9)$ & $167(8)$ & -10 & $-12,-8.3$ & $<0.001$ \\
\hline $\mathrm{VE}_{\mathrm{RCP}}$ & $112(19)$ & $119(16)$ & -7.8 & $-12,-3.7$ & $<0.001$ \\
\hline $\mathrm{RR}_{\mathrm{RCP}}$ & $40(7)$ & $47(11)$ & -7.1 & $-10,-4.1$ & $<0.001$ \\
\hline $\mathrm{O}_{2}$ pulse $_{\mathrm{RCP}}$ & $20.55(4.21)$ & $21.36(2.59)$ & -0.82 & $-1.7,0.08$ & 0.073 \\
\hline $\mathrm{Lac}_{\mathrm{RCP}}$ & $4.31(0.53)$ & $4.40(0.78)$ & -0.09 & $-0.36,0.19$ & 0.5 \\
\hline $\mathrm{VO}_{2} \max$ & $46.9(6.0)$ & $49.9(4.8)$ & -3.0 & $-4.2,-1.8$ & $<0.001$ \\
\hline $\mathrm{VO}_{2} \max _{\mathrm{a}}$ & 3,670 (459) & $3,916(439)$ & -246 & $-343,-149$ & $<0.001$ \\
\hline RERmax & $1.13(0.03)$ & $1.11(0.03)$ & 0.03 & $0.01,0.04$ & $<0.001$ \\
\hline $\mathrm{O}_{2}$ pulse $_{\max }$ & $21.35(2.88)$ & $22.05(2.76)$ & -0.71 & $-1.2,-0.20$ & 0.008 \\
\hline HRmax & $172(8)$ & $178(9)$ & -5.9 & $-7.5,-4.3$ & $<0.001$ \\
\hline VEmax & 157 (25) & $151(22)$ & 5.5 & $1.0,9.9$ & 0.018 \\
\hline RRmax & $57(10)$ & $59(12)$ & -2.7 & $-6.6,-4.9$ & $<0.001$ \\
\hline Lacmax & $10.90(1.65)$ & $9.45(1.82)$ & 1.5 & $0.72,2.2$ & $<0.001$ \\
\hline
\end{tabular}




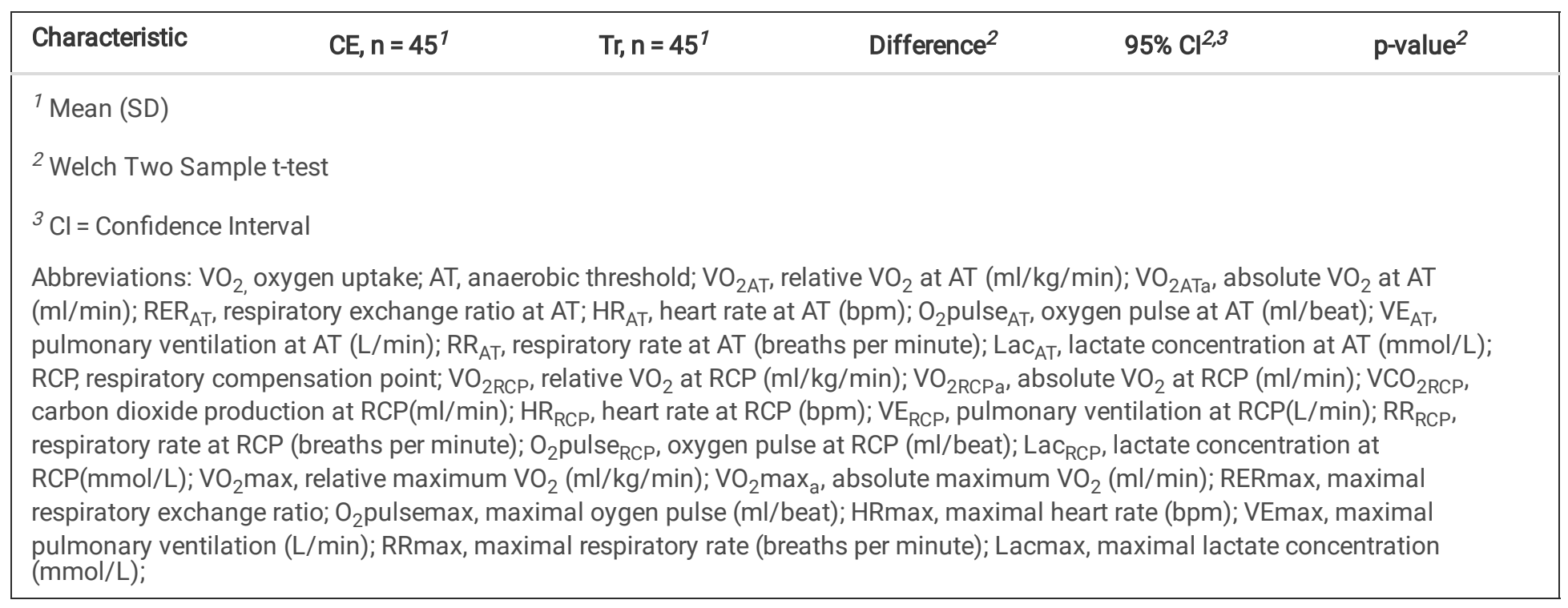


Table 6

Regression analysis for males in two subgroups ( $\leq 40$ years and $>40$ years).

\begin{tabular}{|c|c|c|c|c|c|c|}
\hline $\begin{array}{l}\text { Age } \\
\text { group }\end{array}$ & $\leq 40$ years & & & $>40$ years & & \\
\hline Predictors & BMI & $\mathrm{BF}$ & FFM & $\mathrm{BMI}$ & $\mathrm{BF}$ & FFM \\
\hline \multicolumn{7}{|l|}{$\mathrm{VO}_{2} \mathrm{AT}$} \\
\hline b & 0.02 & 0.15 & 0.01 & -0.92 & 0.39 & 0.16 \\
\hline $95 \% \mathrm{Cl}$ & $\begin{array}{l}-0.94 \\
1.0\end{array}$ & $\begin{array}{l}-0.33 \\
0.63\end{array}$ & $\begin{array}{l}-0.19 \\
0.22\end{array}$ & $\begin{array}{l}-1.7 \\
-0.15\end{array}$ & $\begin{array}{l}0.13 \\
0.64\end{array}$ & $\begin{array}{l}-0.03 \\
0.34\end{array}$ \\
\hline$p$-value & $>0.9$ & 0.5 & $>0.9$ & 0.020 & 0.004 & 0.089 \\
\hline $\mathrm{R}^{2}$ & 0.023 & & & 0.197 & & \\
\hline \multicolumn{7}{|l|}{ HRAT } \\
\hline$b$ & 0.23 & -0.10 & -0.06 & -1.3 & 1.0 & 0.17 \\
\hline $95 \% \mathrm{Cl}$ & $-1.8,2.3$ & $-1.1,0.93$ & $\begin{array}{l}-0.49 \\
0.38\end{array}$ & $\begin{array}{l}-3.5 \\
0.93\end{array}$ & $0.29,1.8$ & $\begin{array}{l}-0.36 \\
0.71\end{array}$ \\
\hline p-value & 0.8 & 0.9 & 0.8 & 0.2 & 0.008 & 0.5 \\
\hline $\mathrm{R}^{2}$ & 0.001 & & & 0.172 & & \\
\hline \multicolumn{7}{|l|}{$\mathrm{VO}_{2} \mathrm{RCP}$} \\
\hline$b$ & -0.16 & 0.30 & 0.05 & -1.4 & 0.80 & 0.15 \\
\hline $95 \% \mathrm{Cl}$ & $\begin{array}{l}-1.1 \\
0.82\end{array}$ & $\begin{array}{l}-0.19 \\
0.80\end{array}$ & $\begin{array}{l}-0.16 \\
0.26\end{array}$ & $\begin{array}{l}-3.1 \\
0.26\end{array}$ & $0.24,1.4$ & $\begin{array}{l}-0.25 \\
0.56\end{array}$ \\
\hline$p$-value & 0.8 & 0.2 & 0.6 & 0.095 & 0.006 & 0.4 \\
\hline $\mathrm{R}^{2}$ & 0.055 & & & 0.169 & & \\
\hline \multicolumn{7}{|l|}{ HRRCP } \\
\hline$b$ & 0.47 & -0.16 & -0.01 & -1.3 & 0.79 & 0.13 \\
\hline $95 \% \mathrm{Cl}$ & $-1.0,1.9$ & $-0.89,0.57$ & $-0.32,0.30$ & $-3.0,0.34$ & $0.24,1.4$ & $-0.27,0.54$ \\
\hline$p$-value & 0.5 & 0.7 & $>0.9$ & 0.11 & 0.006 & 0.5 \\
\hline $\mathrm{R}^{2}$ & 0.008 & & & 0.035 & & \\
\hline \multicolumn{7}{|l|}{$\mathrm{VO}_{2} \max$} \\
\hline b & 0.35 & 0.11 & 0.09 & 1.3 & 0.47 & 0.24 \\
\hline $95 \% \mathrm{Cl}$ & $-0.60,1.3$ & $-0.37,0.59$ & $-0.29,0.12$ & $\begin{array}{l}-2.4 \\
-0.19\end{array}$ & $0.11,0.83$ & $-0.03,0.50$ \\
\hline$p$-value & 0.5 & 0.6 & 0.4 & 0.022 & 0.012 & 0.078 \\
\hline $\mathrm{R}^{2}$ & 0.054 & & & 0.163 & & \\
\hline \multicolumn{7}{|l|}{ HRmax } \\
\hline b & -0.13 & 0.10 & 0.12 & -1.5 & 0.78 & -0.01 \\
\hline $95 \% \mathrm{Cl}$ & $-1.1,0.87$ & $-0.40,0.61$ & $-0.09,0.31$ & $-2.8,-0.16$ & $0.35,1.2$ & $-0.32,0.30$ \\
\hline
\end{tabular}

Abbreviations: BMI, body masa index; $\mathrm{BF}$, body fat (\%); FFM, fat free mass ( $\mathrm{kg})$; $\mathrm{VO}_{2} \mathrm{AT}$, oxygen consumption at anaerobic threshold (ml/kg/min); HRAT, heart rate at anaerobic threshold (bpm); V02RCP, oxygen consumption at respiratory compensation point $(\mathrm{ml} / \mathrm{kg} / \mathrm{min}) ; \mathrm{HRRCP}$, heart rate at respiratory compensation point $(\mathrm{bpm}) ; \mathrm{VO}_{2}$ max, maximum oxygen uptake (ml/kg/min); $\mathrm{HRmax}$, maximal heart rate (bpm). 


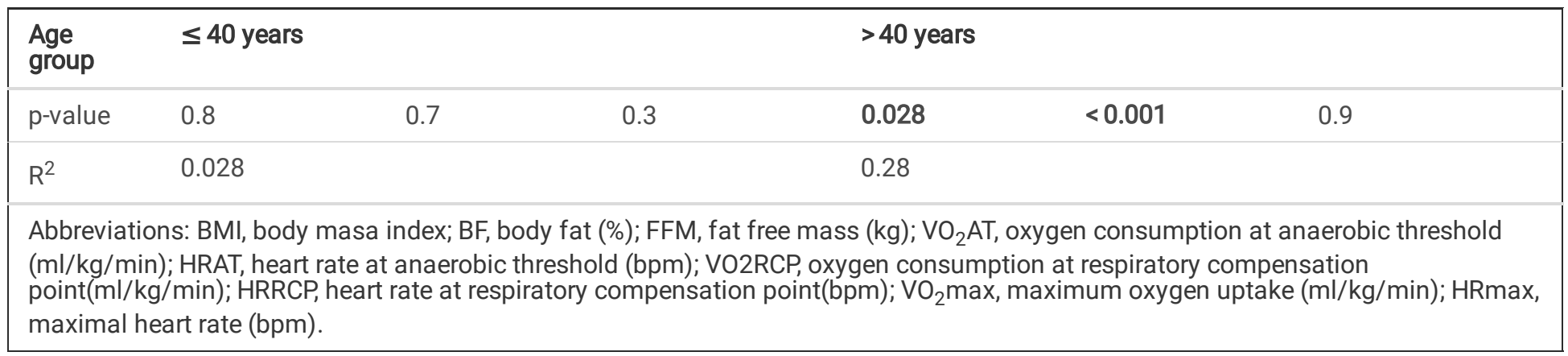

\section{Discussion}

Despite previous research on the topic, it remains unclear whether treadmill and cycle ergometry may be used interchangeably for the monitoring of training in triathletes, largely due to insufficient data on the differences in results obtained from both testing modalities $8,22,36$. In this study we demonstrated significant differences in cardiorespiratory parameters at AT, 2RCP and maximum exertion between cycle ergometry and treadmill testing. Regression models demonstrated significant relationships between $\mathrm{BC}, \mathrm{BMI}$ and training experience, and the differences in $\mathrm{VO}_{2}$ max in cycle ergometry and treadmill testing, especially in the older population of athletes. To the best of our knowledge, this is the first study to evaluate the differences between treadmill and cycle ergometer CPET in a large group of triathletes and the first to analyse factors associated with these differences.

The results of our study show that both male and female triathletes have a significantly higher $\mathrm{VO}_{2} / \mathrm{AT}$ in treadmill than in the cycle ergometer tests. The AT is a crucial parameter in determining performance and in training monitoring in endurance sports, as it indicates the level of exertion an athlete can sustain for a prolonged period of time during competition without rapid lactate build-up ${ }^{8,37}$. Training at the anaerobic threshold (AnT) intensity improves the peak oxygen uptake and the AT level ${ }^{37}$. Recent studies also demonstrate that a large volume of low intensity training (i.e. below the AT) is important for endurance athletes ${ }^{38,39}$.

Previous research was ambiguous as to whether $\mathrm{VO}_{2 \mathrm{AT}}$ differs between testing modalities in triathletes ${ }^{8}$. This contradicts the results of several previous studies which found no differences in $\mathrm{VO}_{2}$ at $\mathrm{AT}$, but were conducted on very small groups (14 participants at most) $8,13,21,24,40$. Some results were similar to our study ${ }^{20}$. The large mean difference in relative $\mathrm{VO}_{2 \mathrm{AT}}$ of $6 \mathrm{ml} / \mathrm{kg} / \mathrm{min}$ in males shown in our study indicates that the values obtained from both testing modalities likely cannot be used interchangeably. A further factor limiting the interchangeability of results from different testing modalities is the large difference in HR at AT of $13 \mathrm{bpm}$ in males and $10 \mathrm{bpm}$ in females, which would hinder the accurate prescription of low intensity training based on HR zones. This is contrary to results from the studies of Hue and Bolognesi, who found differences of $\sim 7 \mathrm{bpm}$, but without statistical significance, perhaps due to limited numbers of participants ${ }^{12,26}$.

We found that the $\mathrm{VO}_{2} / \mathrm{RCP}$ was significantly higher in treadmill than in cycle ergometry both in males and females, although the differences were smaller than in the AT. We also found a large and significant difference in HR at RCP of 7-10bpm, again limiting transferability of results between modalities. To the best of our knowledge, no previous study evaluated RCP in triathletes in treadmill and cycle ergometry testing.

The maximum exertion is the most commonly used parameter to assess the aerobic capacity of athletes ${ }^{8}$. As with the other parameters $\left(\mathrm{VO}_{2} \mathrm{AT}, \mathrm{VO}_{2} \mathrm{RCP}\right)$, we found $\mathrm{VO}_{2}$ max to be significantly higher in treadmill testing than in cycle ergometry. Millet et al. concluded from previous studies that $\mathrm{VO}_{2}$ max is generally similar in treadmill and cycle ergometry testing in triathletes and therefore that triathletes' training adaptation is similar to that of cyclists ${ }^{8}$. This is contrary to the results of our study, most likely due to limited sample sizes. In the present study, the differences in AT were larger than at maximum exertion. This may explain why significant differences were observed more often at AT than at maximum. The $\mathrm{VO}_{2}$ max in our studied population is lower than that reported in most previous studies, probably due to the higher mean age of the participants, which is a known factor limiting $\mathrm{VO}_{2} \mathrm{max}^{8,29}$. HRmax was also significantly higher in treadmill testing than in cycle ergometry in both males and females. The results of previous studies on males were conflicting, some indicating HRmax to be lower in cycle ergometry by $6-10 \mathrm{bpm}{ }^{12,19,20,23}$ and some finding no significant difference ${ }^{13,14,17,22,41}$. Few studies included females and the evidence was also conflicting 24,42 .

We found that the VE was higher in cycle ergometry than on the treadmill, despite a slightly lower ventilation frequency, indicating a higher tidal volume. This is contrary to the lower VE and Vf in cyclists at both AT and RCP. The differences may correspond to higher lactate

Page $16 / 20$ 
accumulation and an acidosis-induced respiratory response in cycle ergometry at maximum, but not at AT and RCP, where lactate levels were similar. This is partly similar to the findings of Koyal et al, who described a higher respiratory response due to higher acidosis in cycle ergometry during low, moderate and high intensity of exercise in untrained subjects ${ }^{43}$. The differences at low and moderate intensity are most likely due to our subjects' experience in triathlon training and therefore lower lactate build-up when cycling at submaximal levels, as it has previously been demonstrated that trained cyclists accumulate less lactate in cycle ergometry ${ }^{10}$. The lower VE and Vf in cycle ergometry at AT and RCP is likely due to the lower $\mathrm{VO}_{2}$.

The variance in differences in $\mathrm{VO}_{2}$ max obtained from treadmill and cycle ergometer testing was significantly explained by BMI, FATP, FFM and training experience in the group $>40$ years of age with an $R^{2}$ of 0.25 . It has previously been shown that $B M$ and $B C$ are related to aerobic capacity and that the physiological ability to consume oxygen is negatively associated with FM ${ }^{44-46}$. However, to the best of our knowledge, this is the first study to demonstrate an association between BC, BMI, and the differences in treadmill and cycle ergometry CPET results. We hypothesize that this relationship may be caused by the physiological differences between cycling and running. Cycling is a non-weight-bearing activity, with far less eccentric activity than during running ${ }^{47}$. Therefore, BM and BC may be more important in treadmill testing than in cycle ergometry. It is unclear why the observed differences are better explained by anthropometric variables in the older group compared to the younger group. We may suspect that the difference in the younger population would be better explained by other factors, perhaps by training volume in cycling or running, which were not evaluated in this study. It is also possible that the differences in BC, especially FFM reflect differences in cardiovascular function, which might play a more important role in older athletes and could have different importance in cycle ergometry and in treadmill testing ${ }^{48,49}$.

Differences in HRmax were significantly explained by BMI and FATP in the older population. It has previously been shown that HRmax is related to $\mathrm{BC}$ and $\mathrm{BMI}$, but it remains unclear what the cause for this relationship is or why $\mathrm{BC}$ is related to the differences in HRmax between treadmill and cycle ergometry ${ }^{50}$.

\section{Conclusions}

$\mathrm{VO}_{2}$ and $\mathrm{HR}$ are higher in treadmill testing at $\mathrm{AT}, \mathrm{RCP}$ and maximum exertion in both male and female triathletes.

The differences are relatively large, which potentially limits the transferability of treadmill and cycle ergometer testing. For optimal fitness evaluation and training planning, both tests should be carried out in triathletes.

The differences are partly explained by BMI, BF, and FFM in the population above 40 years of age.

\section{Study Limitations}

The time intervals between tests varied significantly. Tests were carried out at various times of the season. Training data, except months of experience in triathlon, were unavailable. The female group was too small for meaningful regression analysis, but it was larger than in many previous studies and large enough to observe many significant differences. It was not meaningful to divide females into younger and older age groups, as only 2 females were over 40 years of age. BC was measured with bioelectrical impedance, which may be less accurate than some other methods like CT/MRI BC analysis but is commonly used in sports and therefore may be more practically applicable than more advanced methods.

\section{Declarations}

\section{Author contributions}

SP, SW, and DŚ: conceptualization and resources. JL, SW, DŚ, and AM: investigation. IC, GG, PK: statistical analysis. SP, SW, GG, PK: writing-original draft preparation. SP, SW, DŚ, PK, GG, JL writing - review and editing. AM: supervision. All authors have read and agreed to the published version of the manuscript

\section{Conflicting interests}

$\mathrm{SW}$ is the owner of the sportslab clinic. Other authors have no conflict to declare. 


\section{Data availability}

The datasets generated during and/or analysed during the current study are available from the corresponding author on reasonable request.

\section{References}

1. Chambers, D. J. \& Wisely, N. A. Cardiopulmonary exercise testing-a beginner's guide to the nine-panel plot. BJA Educ, 19, 158-164 (2019).

2. Guazzi, M. et al. EACPR/AHA Scientific Statement. Clinical recommendations for cardiopulmonary exercise testing data assessment in specific patient populations., 126, 2261-2274 (2012).

3. Herdy, A. H. et al. Cardiopulmonary Exercise Test: Background, Applicability and Interpretation. Arq. Bras. Cardiol, 107, 467-481 (2016).

4. Galán-Rioja, M. Ã., González-Mohíno, F., Poole, D. C. \& González-Ravé, J. M. Relative Proximity of Critical Power and Metabolic/Ventilatory Thresholds: Systematic Review and Meta-Analysis. Sports Med, 50, 1771-1783 (2020).

5. Jones, A. M. \& Vanhatalo, A. The 'Critical Power' Concept: Applications to Sports Performance with a Focus on Intermittent HighIntensity Exercise. Sports Med, 47, 65-78 (2017).

6. Löllgen, H. \& Leyk, D. Exercise Testing in Sports Medicine. Dtsch. Arztebl. Int, 115, 409-416 (2018).

7. Hanson, N. et al. Modality determines VO2max achieved in self-paced exercise tests: validation with the Bruce protocol.Eur. J. Appl. Physiol.116, (2016).

8. Millet, G., Vleck, V. \& Bentley, D. Physiological Differences Between Cycling and Running. Sports Med, 39, 179-206 (2009).

9. Costa, M. M. et al. Oxygen consumption and ventilation during constant-load exercise in runners and cyclists. J. Sports Med. Phys. Fitness, 29, 36-44 (1989).

10. Bouckaert, J., Vrijens, J. \& Pannier, J. L. Effect of specific test procedures on plasma lactate concentration and peak oxygen uptake in endurance athletes. J. Sports Med. Phys. Fitness, 30, 13-18 (1990).

11. Basset, F. \& Boulay, M. Specificity of treadmill and cycle ergometer tests in triathletes, runners and cyclists. Eur. J. Appl. Physiol, 81, 214-221 (2000).

12. Hue, O., Le Gallais, D., Chollet, D. \& Préfaut, C. Ventilatory threshold and maximal oxygen uptake in present triathletes. Can. J. Appl. Physiol, 25, 102-113 (2000).

13. Medelli, J. et al. Maximal oxygen uptake and aerobic-anaerobic transition on treadmill and bicycle in triathletes. Jpn. J. Physiol, 43, 347-360 (1993).

14. Zhou, S., Robson, S. J., King, M. J. \& Davie, A. J. Correlations between short-course triathlon performance and physiological variables determined in laboratory cycle and treadmill tests. J. Sports Med. Phys. Fitness, 37, 122-130 (1997).

15. Sleivert, G. G. \& Wenger, H. A. Physiological predictors of short-course triathlon performance. Med. Sci. Sports Exerc, 25, $871-876$ (1993).

16. Miura, H., Kitagawa, K. \& Ishiko, T. Economy during a simulated laboratory test triathlon is highly related to Olympic distance triathlon. Int. J. Sports Med, 18, 276-280 (1997).

17. Kohrt, W. M., Morgan, D. W., Bates, B. \& Skinner, J. S. Physiological responses of triathletes to maximal swimming, cycling, and running. Med. Sci. Sports Exerc, 19, 51-55 (1987).

18. Schabort, E. J., Killian, S. C., Clair Gibson, S., Hawley, A., Noakes, T. D. \& J. A. \& Prediction of triathlon race time from laboratory testing in national triathletes. Med. Sci. Sports Exerc, 32, 844-849 (2000).

19. Hue, O., Le Gallais, D., Chollet, D., Boussana, A. \& Préfaut, C. The influence of prior cycling on biomechanical and cardiorespiratory response profiles during running in triathletes. Eur. J. Appl. Physiol. Occup. Physiol, 77, 98-105 (1998).

20. Kohrt, W. M., O'Connor, J. S. \& Skinner, J. S. Longitudinal assessment of responses by triathletes to swimming, cycling, and running. Med. Sci. Sports Exerc, 21, 569-575 (1989).

21. Albrecht, T. J., Foster, V., Dickinson, A. \& Debever, J. TRIATHLETES: EXERCISE PARAMETERS MEASURED DURING BICYCLE, SWIM BENCH, AND TREADMILL TESTING.Med. Sci. Sports Exerc.18, (1986).

22. Basset, F. \& Boulay, M. Treadmill and Cycle Ergometer Tests are Interchangeable to Monitor Triathletes Annual Training. J. Sports Sci. Med, 2, 110-116 (2003)

Page $18 / 20$ 
23. Roecker, K., Striegel, H. \& Dickhuth, H. H. Heart-Rate Recommendations: Transfer Between Running and Cycling Exercise? Int. J. Sports Med, 24, 173-178 (2003).

24. Schneider, D. A., Lacroix, K. A., Atkinson, G. R., Troped, P. J. \& Pollack, J. Ventilatory threshold and maximal oxygen uptake during cycling and running in triathletes. Med. Sci. Sports Exerc, 22, 257-264 (1990).

25. Galy, O., Le Gallais, D., Hue, O., Boussana, A. \& Préfaut, C. Is exercise-induced arterial hypoxemia in triathletes dependent on exercise modality? Int. J. Sports Med, 26, 719-726 (2005).

26. Bolognesi, M. Ventilatory Threshold and Maximal Oxygen Uptake During Cycling and Running in Duathletes(1997).

27. O’Toole, M. L., Hiller, D. B., Crosby, L. O. \& Douglas, P. S. The ultraendurance triathlete: a physiological profile. Med. Sci. Sports Exerc, $19,45-50$ (1987).

28. Martin-Rincon, M. \& Calbet, J. A. L. Progress Update and Challenges on V. O(2max) Testing and Interpretation. Front. Physiol, 11, 1070 (2020).

29. Kaminsky, L. A., Arena, R. \& Myers, J. Reference Standards for Cardiorespiratory Fitness Measured With Cardiopulmonary Exercise Testing: Data From the Fitness Registry and the Importance of Exercise National Database. Mayo Clin. Proc. 90, 1515-1523(2015).

30. Beaver, W. L., Wasserman, K. \& Whipp, B. J. A new method for detecting anaerobic threshold by gas exchange. J. Appl. Physiol, 60, 2020-2027 (1986).

31. Team, R. C. R: A language and environment for statistical computing(2013).

32. Daniel, D. et al. E. C. Z. gtsummary: Presentation-Ready Data Summary and Analytic Result Tables. R package version 1.4.2(2021).

33. Stekhoven, D. J. \& missForest Nonparametric Missing Value Imputation using Random Forest. R package version 1.4(2013).

34. Fleg, J. L. \& Lakatta, E. G. Role of muscle loss in the age-associated reduction in VO2 max. J. Appl. Physiol, 65, 1147-1151 (1988).

35. Ades, P. A. \& Toth, M. J. Accelerated decline of aerobic fitness with healthy aging: what is the good news? Circulation vol. 112 624626(2005).

36. Carey, D. G., Tofte, C., Pliego, G. J. \& Raymond, R. L. Transferability of running and cycling training zones in triathletes: implications for steady-state exercise. J. strength Cond. Res, 23, 251-258 (2009).

37. Ghosh, A. K. Anaerobic threshold: its concept and role in endurance sport. Malays. J. Med. Sci, 11, 24-36 (2004).

38. Bourgois, J. G., Bourgois, G. \& Boone, J. Perspectives and determinants for training-intensity distribution in elite endurance athletes. Int. J. Sports Physiol. Perform, 14, 1151-1156 (2019).

39. Casado, A., Hanley, B., Santos-Concejero, J. \& Ruis-Pérez, L. M. World-class long-distance running performances are best predicted by volume of easy runs and deliberate practice of short interval and tempo runs.J. strength Cond. Res.(2019).

40. Kreider, R. B., Boone, T., Thompson, W. R., Burkes, S. \& Cortes, C. W. Cardiovascular and thermal responses of triathlon performance. Med. Sci. Sports Exerc, 20, 385-390 (1988).

41. Bassett, D. R. \& Howley, E. T. Limiting factors for maximum oxygen uptake and determinants of endurance performance. Med. Sci. Sports Exerc, 32, 70-84 (2000).

42. Millet, G., Vleck, V. \& Bentley, D. Physiological requirements in triathlon.J. Hum. Sport Exerc.6, (2011).

43. Koyal, S. N., Whipp, B. J., Huntsman, D., Bray, G. A. \& Wasserman, K. Ventilatory responses to the metabolic acidosis of treadmill and cycle ergometry. J. Appl. Physiol, 40, 864-867 (1976).

44. Baker, J. S., Bailey, D. M. \& Davies, B. The relationship between total-body mass, fat-free mass and cycle ergometry power components during 20 seconds of maximal exercise. J. Sci. Med. Sport, 4, 1-9 (2001).

45. Minasian, V., Marandi, S. M., Kelishadi, R. \& Abolhassani, H. Correlation between aerobic fitness and body composition in middle school students. Int. J. Prev. Med, 5, S102 (2014).

46. Vargas, V., Lira, C., Vancini, R., Rayes, A. \& Andrade, M. Fat mass is negatively associated with the physiological ability of tissue to consume oxygen.Mot. Rev. Educ. Física24, (2018).

47. Nicol, C., Komi, P. \& Marconnet, P. Fatigue effects of marathon running on neuromuscular performance: II. Changes in force, integrated electromyographic activity and endurance capacity. Scand. J. Med. Sci. Sport. - SCAND J MED SCI Sport, 1, 18-24 (2007).

48. Zeng, Q., Sun, X. N., Fan, L. \& Ye, P. Correlation of body composition with cardiac function and arterial compliance. Clin. Exp. Pharmacol. Physiol, 35, 78-82 (2008).

49. Carrick-Ranson, G. et al. The Effect of Age-related Differences in Body Size and Composition on Cardiovascular Determinants of V02max. Journals Gerontol. Ser. A, 68, 608-616 (2013). 


\section{Figures}
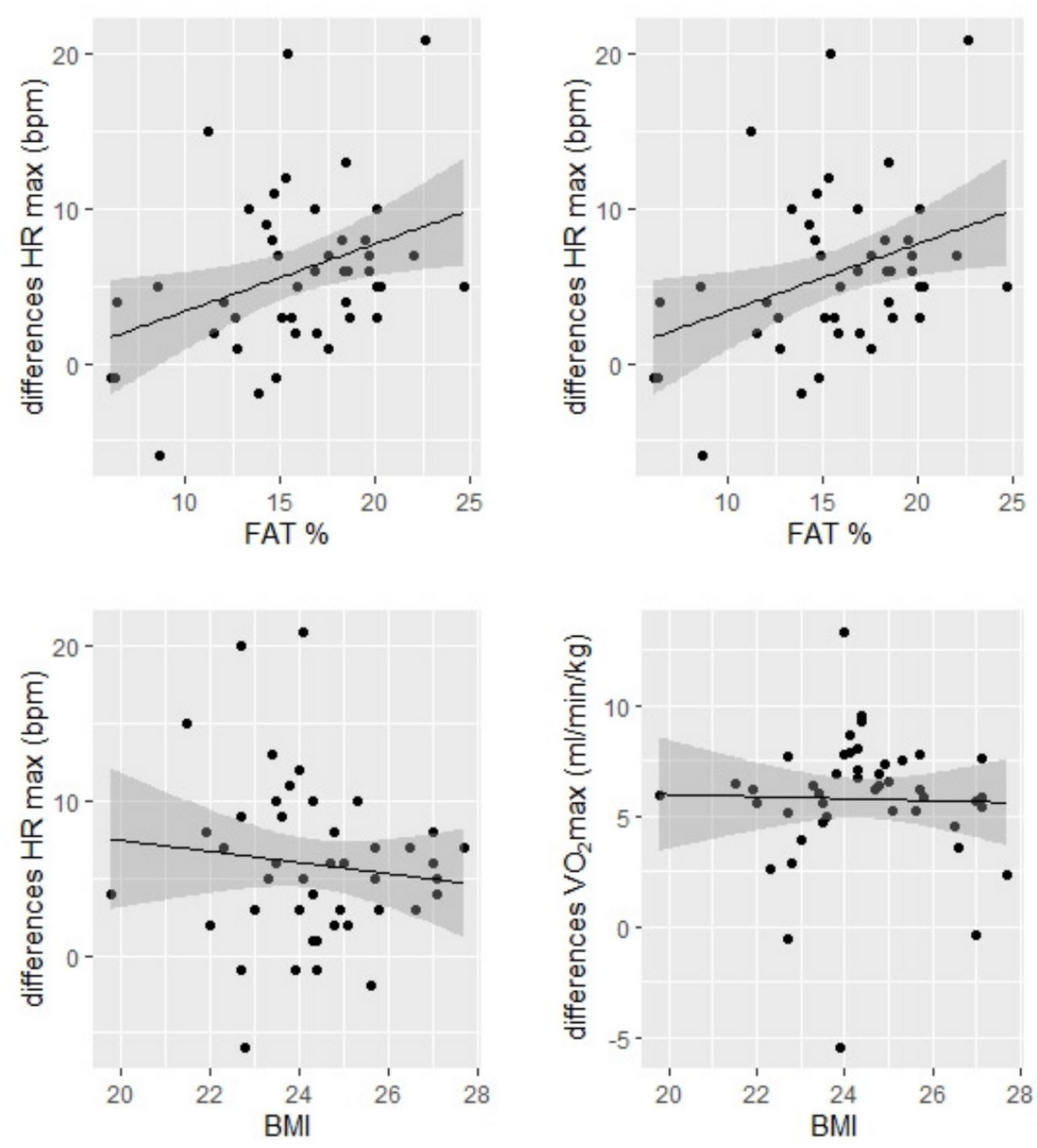

\section{Figure 1}

Regression analysis for males in subgroup $>40$ years. Legend: Multiple linear regression was performed to evaluate the association between differences in treadmill and cycle ergometer test results, and BMI, body fat and fat free mass in amateur male triathletes. The figure presents the most important (highest R2) relationships in the group of males >40years of age $(n=40)$ as linear regression graphs. Abbreviations: BMI, body mass index $\left(\mathrm{kg} / \mathrm{m}^{2}\right)$; HR max, maximal heart rate (bpm); VO囚 max, maximum oxygen uptake (ml/min/kg). 